\section{References}

1 HM Courts and Tribunal Service. Mental Health Tribunals Guidance. HM Courts and Tribunal Service, no date (http://www.justice.gov.uk/ guidance/courts-and-tribunals/tribunals/mental-health/index.htm).

2 Davison P, Perez de Albeniz P. Reports prepared for the Mental Health Review Tribunals and Managers' Reviews. Psychiatr Bull 1997; 21: 364-6.

3 Ismail K, Smith S, Maden T. Mental health review tribunal medical reports. Psychiatr Bull 1998; 22: 615-8.

4 Regional Chairmen of the Mental Health Review Tribunal for England and Wales Guidance for the Preparation of Medical Reports for the MHRT. Mental Health Review Tribunal Secretariat, 2000.

5 Egleston $\mathrm{P}$, Hunter MD. Improving the quality of medical reports to mental health review tribunals. Psychiatr Bull 2002; 26: 215-8.
6 O'Muirithe B, Shankar R. An audit of the quality of reports to Mental Health Review Tribunals. Med Sci Law 2008; 48: 221-4.

7 HM Courts and Tribunal Service. History of Tribunals Reforms. HM Courts and Tribunal Service, no date (http://www.justice.gov.uk/downloads/ about/hmcts/tribunals/Tribunals-History.pdf).

8 HM Courts and Tribunal Service. Reports for Mental Health Tribunals. Reports Guidance Booklet. Tribunals Service, 2010.

9 Webster CD, Douglas KS, Eaves D, Hart SD. HCR-20: Assessing Risk for Violence (Version 2). Simon Fraser University, 1997.

10 Department of Health. The Journey to Recovery - The Government's Vision for Mental Health Care. Department of Health, 2001.

11 HM Courts and Tribunal Service. Reports for Mental Health Tribunals (2nd edn). Reports Guidance Booklet. Tribunals Service, 2012.

\title{
Audit of monitoring of the parameters of metabolic syndrome in patients on clozapine
}

\author{
John Tully, ${ }^{1}$ Christina Sim, ${ }^{1}$ Razi Hemani, ${ }^{1}$ Malik Munir, ${ }^{1}$ Nasir Khalil, ${ }^{1}$ Sabina Fahy ${ }^{1}$
}

The Psychiatrist (2012), 36, 466-469, doi: 10.1192/pb.bp.111.037994

${ }^{1}$ St Brigid's Hospital, Ballinasloe, Ireland Correspondence to John Tully (john.tully@slam.nhs.uk)

First received 28 Nov 2011, final revision 26 Jul 2012, accepted 13 Aug 2012

\begin{abstract}
Aims and Method High rates of metabolic syndrome exist among patients on clozapine. Monitoring its parameters facilitates interventions which may alleviate negative health consequences. We completed an audit of the monitoring of the parameters of metabolic syndrome in patients on clozapine. The results were compared with the Maudsley Guidelines for monitoring in patients on any antipsychotic medication.
\end{abstract}

Results Initial audit showed high overall rates of concordance with guidelines for the frequency of measurement of blood pressure (91.8\%), but much lower rates for measuring fasting blood glucose (43.2\%) and lipid profile $(52.7 \%)$, and no record of analysis of waist circumference. This prompted development of a formal protocol for measuring parameters. Repeat audit after 1 year showed marked improvement in rates of measurement.

Clinical implications Implementation of relatively straightforward measures, such as the introduction of a one-page form on which to record parameters, can lead to a much improved rate of monitoring for metabolic syndrome. This should in turn prompt therapeutic interventions, which are discussed.

Declaration of interest None.
People with major mental illnesses have an increased prevalence of being overweight and obese, and having hyperglycaemia, dyslipidaemia and hypertension compared with the general population. ${ }^{1}$ These parameters make up the basis of metabolic syndrome as defined by the National Cholesterol Education Programme (NCEP). ${ }^{2}$ Metabolic syndrome in turn predicts development of cardiovascular disease and diabetes. ${ }^{3,4}$ It has been shown that secondgeneration antipsychotics contribute to the development of the syndrome. ${ }^{1,5,6}$ Clozapine is particularly implicated in this regard due to the fact that it has been shown, along with olanzapine, to have the greatest weight gain potential, ${ }^{7}$ and that the risk of diabetes appears to be higher for clozapine than for other second-generation antipsychotics. ${ }^{8}$ One major US study showed a prevalence of metabolic syndrome in patients on clozapine of $53.8 \%$ compared with $20.7 \%$ of those in a comparison group, ${ }^{9}$ while an Irish study showed a comparatively high rate of $46.4 \%$ in its cohort. ${ }^{10}$

Monitoring of the parameters of metabolic syndrome facilitates interventions which may alleviate the negative health consequences of the syndrome. ${ }^{11}$ It has also been shown to be an effective and cost-effective intervention. ${ }^{12}$ 
Several guidelines exist regarding the recommended frequency of monitoring of these parameters. These include the Maudsley Guidelines ${ }^{13}$ and the recommendations of the Mount Sinai Conference. ${ }^{14}$

We proposed to audit the monitoring of parameters of metabolic syndrome (as defined by NCEP) in patients on clozapine in East Galway Mental Health Service, Ireland, as compared to the Maudsley Guidelines for frequency of monitoring of these parameters in patients on antipsychotic medication. For waist circumference and lipids (triglycerides and high-density lipoprotein), it is recommended that these be measured at baseline, frequently for 3 months, and then yearly. Fasting plasma glucose should be taken at baseline, at 4-6 months, and then yearly. Blood pressure should be taken at baseline, and frequently during dose titration.

\section{Method}

This audit was designed by the authors following a meeting of the Audit Committee in St Brigid's Hospital, Ballinasloe, Ireland. Ethical approval was sought and obtained from St Brigid's Senior Management Committee.

A checklist was devised using the parameters for metabolic syndrome recommended by the NCEP guidelines. The checklist was completed in January/February 2010 on a sample of patients on clozapine in East Galway Mental Health Service, using patient information records over the preceding 1-year period (calendar year 2009).

It was initially planned to include all patients on clozapine within the service. Unfortunately, owing to time and resource constraints, this did not prove to be feasible. The service is spread out throughout a wide geographical area and in some instances charts were misfiled or unavailable (e.g. some files had been taken to clinics elsewhere or into an in-patient unit). A small number of isolated community settings with small numbers of patients were not accessed. This audit had been identified to be completed within a fixed time frame for local audit procedures.

We decided to follow through with the number of charts reviewed at the time of deadline for the first phase of audit. We ensured that at least $50 \%$ of patients within each sector area had been included, so as not to skew the sample by inclusion of a disproportionate number from a sector area which was performing particularly well or poorly.
In February/March 2011, the audit was repeated using an identical checklist for the calendar year 2010. At this point, there were 113 patients on clozapine within the service. It was found that the standardised monitoring form was being used in $92 \%$ of all cases across the service $(n=104)$. However, we believed that using a sample of the same size as used in phase one of the audit for comparison of data would be most appropriate. Only the first 74 charts reviewed at the second phase of audit were thus included in data analysis, again ensuring at least $50 \%$ of charts from each sector area were included.

A protocol for intervention following the first phase of audit was devised. The details of this protocol are as follows:

- use of a standardised form for documentation of the parameters of metabolic syndrome as specified by NCEP

- development of a standardised protocol for dealing with abnormal results across the East Galway service

- re-audit of rates of measurement in 1 year

- ongoing audit and re-education on this topic.

All data were compiled and then analysed using SPSS version 18 for Windows.

\section{Results}

The sample from the first phase of audit was comprised of 50 males (67.5\%; mean age 36.5 years, range 20-64) and 34 females (32.5\%; mean age 41.2 years, range $24-60)$. The sample from the second phase was comprised of 54 males (72.9\%; mean age 34.7 years, range $20-59)$ and 20 females (27.1\%; mean age 39.6 years, range $24-58$ ).

Table 1 gives an overview of results for frequency of measurement of each individual parameter in accordance with guidelines over a period of 1 year. Although high overall rates of accordance with recommended frequency were seen for measurement of blood pressure (91.8\%) and weight $(83.8 \%)$, lower rates were seen in the parameters of fasting blood glucose (43.2) and triglyceride and high-density lipoprotein (lipid profile) (52.7\%).

In the second phase of audit, there were statistically significant improvements between samples in rates of measurement in accordance with guidelines for lipid profile (70.3\%, $P=0.042)$ and fasting blood glucose $(64.8 \%$, $P=0.013)$. Waist circumference, which had not been previously recorded for any patient, was documented in $97.9 \%$ of records sampled $(P<0.0001)$.

\begin{tabular}{|c|c|c|c|}
\hline Metabolic syndrome parameter & $\begin{array}{l}\text { Number measured } \\
\text { in accordance with guidelines } \\
\text { on initial audit (\%) }\end{array}$ & $\begin{array}{c}\text { Number measured } \\
\text { in accordance with guidelines } \\
\text { on repeat after } 1 \text { year }(\%)\end{array}$ & $P^{a}$ \\
\hline Blood pressure & $68(91.8)$ & $72(97.2)$ & 0.2754 \\
\hline Weight & $62(83.8)$ & $\mathrm{n} / \mathrm{a}^{\mathrm{b}}$ & $\mathrm{n} / \mathrm{a}^{\mathrm{b}}$ \\
\hline Lipid profile (high-density lipoprotein and triglycerides) & $39(52.7)$ & $52(70.3)$ & 0.0422 \\
\hline Fasting glucose & $32(43.2)$ & $48(64.8)$ & 0.0131 \\
\hline
\end{tabular}

$\mathrm{n} / \mathrm{a}$, not applicable.

a. $P$-values calculated using two-tailed Fisher's exact test with $2 \times 2$ contingency table

b. Weight and body mass index were not defined as required parameters following initial audit. 


\section{Discussion}

People with major mental illnesses have an increased prevalence of metabolic syndrome, as defined by NCEP. ${ }^{1}$ Patients on clozapine are particularly at risk in this regard ${ }^{7,8}$ and warrant especially close monitoring of the parameters of metabolic syndrome to facilitate timely interventions. Metabolic syndrome is a relatively novel concept in medicine and there has been considerable discussion about how best to define and measure its existence. ${ }^{15}$ The NCEP guidelines ${ }^{2}$ and those of the International Diabetes Federation (IDF) ${ }^{15}$ have become the most widely accepted in clinical practice. Following consultation with a number of local endocrinology teams, the NCEP guidelines were chosen for this audit, on the basis that these are more commonly used in Ireland.

In terms of implications for the service, adequate screening for metabolic syndrome allows for measurements outside of the normal range to be addressed by such interventions. Interventions recommended for metabolic syndrome can be broadly divided into two groups: lifestyle modification and pharmacological treatment. The Report of the National Heart, Lung, and Blood Institute/American Heart Association Conference on Scientific Issues Related to Definition ${ }^{11}$ acknowledged that clinical management should focus first on lifestyle changes, particularly weight reduction and increased exercise. A 2003 study found that out of 105 participants with metabolic syndrome at baseline, $30.5 \%$ were no longer classified as having the syndrome after 20 weeks of supervised aerobic exercise training. ${ }^{16}$ The most obvious benefit was to triglyceride levels, with $43 \%$ showing improvement. Fasting plasma glucose and insulin resistance of $91 \%$ of participants did not improve however. ${ }^{16}$ Also of note, restriction of the overall dietary carbohydrate intake is more effective in reducing the most common symptoms of metabolic syndrome than the more commonly prescribed reduction in dietary fat intake. ${ }^{17}$ This evidence suggests that simple advice on diet and exercise should be a cornerstone of management of patients with metabolic syndrome within our service.

In terms of pharmacological interventions, abnormal lipid profile can be addressed with a number of treatments. Management of elevated low-density lipoprotein includes consideration of all statins at all indicated ranges and the choice of drug and dose should be individualised to the patient and titrated to achieve guideline-recommended goals. ${ }^{18}$ Management of reduced high-density lipoprotein remains controversial, but starts with diet/exercise modifications and may include niacin; certain statins may help, but this is not yet a widely accepted indication. ${ }^{18}$ Fibrates have been shown to reduce end-points of cardiovascular disease in patients with atherogenic dyslipidaemia and metabolic syndrome ${ }^{19}$ and may serve as an important adjunct in overweight patients with elevated triglyceride and low high-density lipoprotein cholesterol. ${ }^{20}$ The use of aspirin to treat prothrombotic state in metabolic syndrome has also been identified as promising. ${ }^{20}$ Metformin has been used for many years for the treatment of type 2 diabetes. More recently, treatment with thiazolidinediones has been introduced. Both of these treatments have been identified as being potentially beneficial for metabolic syndrome. There are, however, currently no studies on cardiovascular end-points in patients with metabolic syndrome treated with metformin or thiazolidinediones. ${ }^{18}$ Thus, routine referral in accordance with screening for and management of diabetes should be the target here. This also applies for hypertension. These drug therapies can be introduced in the primary or secondary care setting and the onus is on our service to ensure abnormal measures are identified and appropriately treated.

The first phase of audit highlighted limitations in practice on monitoring, namely:

- there was no formal protocol for measurement of the parameters of metabolic syndrome in patients on clozapine in the East Galway Mental Health Service;

- clinical practice was not seen to be taking into account the important factor of central obesity at the time of initial audit, with waist circumference not documented in any case.

To address this problem, it was agreed that a standardised form developed for measurement of parameters of metabolic syndrome be used in all patients on clozapine in the service. These forms were to be filed in each patient's medical notes. Following implementation of a new protocol, the monitoring form had been used in $92 \%$ of cases. Waist circumference was shown to be measured in accordance with guidelines in a high proportion (97.2\%) of patients sampled in the second phase of audit. There were also statistically significant improvements in rates of measurement for fasting glucose and lipid profile.

These were encouraging developments which suggested that straightforward educational and practical interventions can lead to significant improvements in practice, and should serve to continue to improve practice in this area. It is also worth noting that the monitoring form introduced as part of the protocol was a brief document which on the basis of separate feedback was not regarded as cumbersome or time consuming by staff. It was concluded that the monitoring of metabolic syndrome in patients on clozapine warranted ongoing review and further audit was planned on an annual basis. It was also agreed that a similar system for monitoring be implemented in due course for all patients on second-generation antipsychotics.

Improvements were shown to be limited by some inconsistencies in documentation and initially by nondevelopment of formal procedures in dealing with abnormal results. These issues highlighted the need for ongoing educational interventions in this area. Our study was further limited by a relatively small sample size.

\section{About the authors}

John Tully, Registrar in Psychiatry of Later Life, Christina Sim, Registrar in Psychiatry of Later Life, Razi Hemani, Registrar in Psychiatry, Malik Munir, Registrar in Psychiatry, Nasir Khalil, Registrar in Psychiatry of Later Life, Sabina Fahy, Consultant in Psychiatry of Later Life, all at St Brigid's Hospital, Ballinasloe, Co. Galway, Ireland.

\section{References}

1 Newcomer JW. Metabolic syndrome and mental illness. Am J Manag Care 2007; 13: S170-7. 
2 National Cholesterol Education Program (NCEP) Expert Panel on Detection, Evaluation, and Treatment of High Blood Cholesterol in Adults (Adult Treatment Panel III). Third report of the National Cholesterol Education Program (NCEP) Expert Panel on Detection, Evaluation, and Treatment of High Blood Cholesterol in Adults (Adult Treatment Panel III). Final report. Circulation 2002; 106: 3143-421.

3 Lakka HM, Laaksonen DE, Lakka TA, Niskanen LK, Kumpusalo E, Tuomilehto J, et al. The metabolic syndrome and total and cardiovascular disease mortality in middle-aged men. JAMA 2002; 288: 2709-16.

4 Ford ES. Risks for all-cause mortality, cardiovascular disease, and diabetes associated with the metabolic syndrome: a summary of the evidence. Diabetes Care 2005; 28: 1769-78

5 Casey DE, Haupt DW, Newcomer JW, Henderson DC, Sernyak MJ, Davidson $M$, et al. Antipsychotic-induced weight gain and metabolic abnormalities: implications for increased mortality in patients with schizophrenia. J Clin Psychiatry 2004; 65 (suppl 7): 4-18.

6 Newcomer JW. Second-generation (atypical) antipsychotics and metabolic effects: a comprehensive literature review. CNS Drugs 2005; 19 (suppl 1): 1-93.

7 Allison DB, Mentore JL, Heo M, Chandler LP, Cappelleri JC, Infante MC, et al. Antipsychotic-induced weight gain: a comprehensive research synthesis. Am J Psychiatry 1999; 156: 1686-96.

8 Lund BC, Perry P, Brooks JM, Arndt S. Clozapine use in patients with schizophrenia and the risk of diabetes, hyperlipidaemia and hypertension: a claims based approach. Arch Gen Psychiatry 2001; 58: 1172-6.

9 Lamberti JS, Olson D, Crilly JF, Olivares T, Williams GC, Tu X, et al. Prevalence of the metabolic syndrome among patients receiving clozapine. Am J Psychiatry 2006; 163: 1273-6.

10 Ahmed M, Hussain I, O'Brien SM, Dineen B, Griffin D, McDonald C. Prevalence and associations of the metabolic syndrome among patients prescribed clozapine. Ir J Med Sci 2008; 177: 205-10.
11 Grundy SM, Brewer Jr HB, Cleeman JI, Smith Jr SC, Lenfant C. Definition of metabolic syndrome: Report of the National Heart, Lung, and Blood Institute/American Heart Association conference on scientific issues related to definition. Circulation 2004; 109: 433-8.

12 Straker DO, Correll CU, Kramer-Ginsberg E, Abdulhamid N, Koshy F,

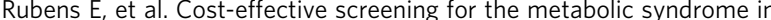
patients treated with second-generation antipsychotic medications. Am J Psychiatry 2005; 162: 1217-21.

13 Taylor D, Paton C, Kerwin R. The Maudsley Prescribing Guidelines (9th edn). Taylor \& Francis, 2007.

14 Covell NH, Jackson CT, Weissman EM. Health monitoring for patients who have schizophrenia. Summary of the Mount Sinai Conference recommendations. Postgrad Med 2006; Spec No: 20-6.

15 Alberti KG, Zimmet P, Shaw J. The metabolic syndrome - a new worldwide definition. A Consensus Statement from the International Diabetes Federation. Diabet Med 2006; 23: 469-80.

16 Katzmaryk PT, Leon AS, Wilmore JH, Skinner JS, Rao DC, Rankinen T, et al. Targeting the metabolic syndrome with exercise: evidence from the HERITAGE Family Study. Med Sci Sports Exerc 2003; 35: 1703-9.

17 Volek JS, Feinman RD. Carbohydrate restriction improves the features of metabolic syndrome. metabolic syndrome may be defined by the response to carbohydrate restriction. Nutr Metab (Lond) 2005; 2: 31

18 Wang SS, Subhi Ali Y, Pearlman JD, Talavera F. Metabolic syndrome. Medscape Reference, 2012. Available online at: http:// emedicine.medscape.com/article/165124-overview.

19 Rubins HB. Triglycerides and coronary heart disease: implications of recent clinical trials. J Cardiovasc Risk 2000; 7: 339-45.

20 Barter PJ, Rye KA. Is there a role for fibrates in the management of dyslipidemia in the metabolic syndrome? Arterioscler Thromb Vasc Biol 2008; 28: 39-46.

21 Shields TM, Hennekens $\mathrm{CH}$. Management of metabolic syndrome: aspirin. Endocrinol Metab Clin North Am 2004; 33: 577-93. 